\title{
A study of modal strain energy change ratio method for the damage identification of functionally graded Euler-Bernoulli beam
}

\author{
Lixin Huang ${ }^{1, a *}$, Shiyan, Yue ${ }^{1, b}$, Zhongming $\mathrm{Hu}^{1, \mathrm{c}}$ and Zhenzhen Yang ${ }^{2, \mathrm{~d}}$ \\ ${ }^{1}$ School of Civil Engineering, Guangxi University, Nanning 530004, China \\ ${ }^{2}$ School of Civil Engineering, Xuchang University, Xuchang 461000, China \\ agxuhuanglixin@163.com, b983260916@qq.com, 1164549746@qq.com, d809689594@qq.com
}

Keywords: Damage identification; Modal strain energy; Functionally graded Euler-Bernoulli beam; Finite element method.

Abstract. An identification method based on modal strain energy is proposed to identify the damage of a functionally graded (FG) Euler-Bernoulli beam. Based on the strain energy of the FG Euler-Bernoulli beam, the expression of element modal strain energy (EMSE) is developed for the two-node beam element with three degrees of freedom per node. The finite element method (FEM) is employed to compute the element stiffness matrix and the modal parameters of the beam. The EMSE is calculated by using the FEM results. On this basis, the damage indicator for the FG Euler-Bernoulli beam is defined by way of the change of the EMSE before and after the occurrence of damage. A numerical example is given to show the validity of the proposed method for the damage identification of the FG Euler-Bernoulli beam. Numerical results reveal that the proposed method has very good capability for identifying damage in the FG Euler-Bernoulli beam and is robust to the effects of measurement noise.

\section{Introduction}

As a new kind of inhomogeneous composite materials, functionally graded materials (FGMs) have been the subject of considerable research in recent years. The properties of FGMs vary continuously in space, which makes FGMs tailored to a broad range of applications and working environments via the design of the material gradients. FGMs beam-like structures, which are very important structural components in engineering, have been increasingly used in many fields, such as aerospace, automotive engineering, chemical engineering, etc., due to their unique graded feature. In reality, damage often takes place in the FGMs beam-like structures, which will reduce the system safety performance. The accumulating continually damage is dangerous for the structures in the service life because this damage is usually unnoticed and results in a catastrophic structural failure. Therefore, considerable research is necessarily undertaken to identify the damage in the FGMs beam-like structures for FGMs application in modern engineering.

The physical properties of a structure, such as mass, damping, stiffness, will be modified when the damage occurs in the structure[1]. These modifications will induce the changes in the modal parameters such as natural frequencies, mode shapes of the structure. Therefore, the modal parameters have been widely used to identify the existence, the location and the extent of damage. Naturally, vibration-based damage identification techniques (VBDIT) have been developed extensively for the reliable and efficient damage identification. Doebling et al.[2] provided a more thorough review of VBDIT for detecting, localizing, and characterizing damages in structures by examining changes in measured modal parameters. Strains are more sensitive to a small defect in a structure than displacements. Hence, more attention has been paid to the damage identification techniques using shape derivatives or strain energy[3-9].

Damage identification method which is based on modal strain energy (MSE) has superior in many respects such as the sensitivity to local defects, severity estimation, stability and noise resistance ability[5-9]. Shi et al.[6] proposed the modal strain energy change ratio method (MSECRM) to localize structural damage for the isotropic beam. Their method has higher sensitivity to local damage

* Corresponding author 
and very good anti-noise property for identifying damage in the isotropic beam. The main objective of this paper is to study the damage identification of an functionally graded (FG) Euler-Bernoulli beam based on the MSECRM. The expression of element modal strain energy (EMSE) is developed for the two-node beam element with three degrees of freedom per node and then the damage indicator for the damage identification of the beam is obtained by way of modal strain energy change ratio. A numerical example is given to demonstrate the effectiveness of the proposed method.

\section{Material Properties of FG Euler-Bernoulli Beam}

Fig.1 shows an FG Euler-Bernoulli simply-supported beam composed of ceramic and metal components. The beam has a rectangular cross section of length $L$, width $b$ and thickness $h$. The upper surface component is pure ceramic and the lower surface component is pure metal. It is assumed that the material properties of the beam such as Young's modulus $E$ and mass density $\rho$ vary continuously through the beam thickness according to power-law form[10]. Therefore, the material properties can be represented as the following function of the coordinate $z$ :

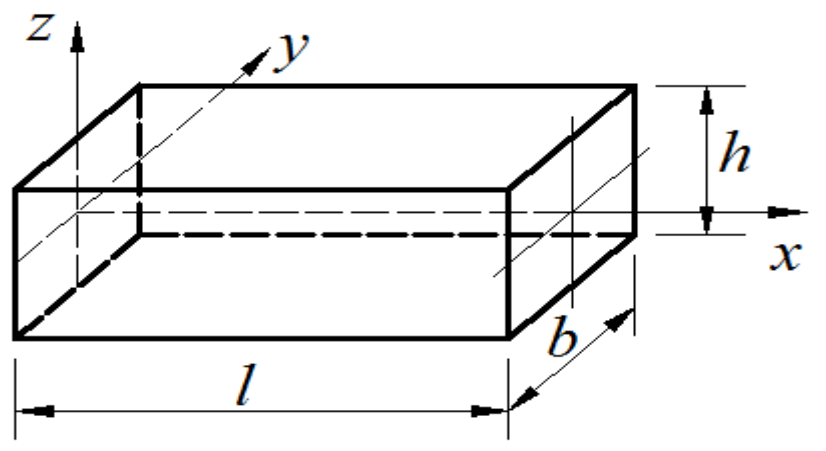

Fig. 1 A functionally graded Euler-Bernoulli beam

$$
P(z)=P_{m}+\left(P_{c}-P_{m}\right)\left(\frac{z}{h}+\frac{1}{2}\right)^{k} \quad-\frac{h}{2} \leq z \leq \frac{h}{2} .
$$

where $P_{c}$ and $P_{m}$ are the values of the material properties of the ceramic and metal components of the FG Euler-Bernoulli beam respectively, and $k$ is the non-negative power-law exponent denoting the material nonhomogeneity through the thickness of the beam.

\section{The EMSE of FG Euler-Bernoulli Beam}

According to the theory of Euler-Bernoulli beam, the expressions of the displacements, strains and stresses are given as

$$
\begin{aligned}
& \left\{\begin{array}{l}
u(x, z, t) \\
u(x, z, t)
\end{array}\right\}=\left[\begin{array}{ccc}
1 & 0 & -z \\
0 & 1 & 0
\end{array}\right]\left\{\begin{array}{l}
u_{0}(x, t) \\
w_{0}(x, t) \\
\frac{\partial w_{0}(x, t)}{\partial x}
\end{array}\right\} . \\
& \varepsilon_{x x}=\frac{\partial u(x, z, t)}{\partial x}=\left[\begin{array}{ll}
1 & -z
\end{array}\right]\left\{\begin{array}{l}
\frac{\partial u_{0}(x, t)}{\partial x} \\
\frac{\partial^{2} w_{0}(x, t)}{\partial x^{2}}
\end{array}\right\} .
\end{aligned}
$$




$$
\sigma_{x x}=E(z) \varepsilon_{x x}=E(z)\left[\begin{array}{ll}
1 & -z
\end{array}\right]\left\{\begin{array}{c}
\frac{\partial u_{0}(x, t)}{\partial x} \\
\frac{\partial^{2} w_{0}(x, t)}{\partial x^{2}}
\end{array}\right\} .
$$

where $u_{0}$ and $w_{0}$ denote respectively the axial and the transverse displacement of any point on the mid-plane, and $t$ is time.

Assume the beam is divided into $n$ elements. The total strain energy of the beam can be expressed as

$$
U=\int_{V} \frac{1}{2} \sigma_{x x}^{T} \varepsilon_{x x} d v=\sum_{j=1}^{n} U_{j} .
$$

where $U_{j}$ is the strain energy of the $j$ th element, i.e.,

$$
U_{j}=\int_{V_{j}} \frac{1}{2} \sigma_{x x}^{T} \varepsilon_{x x} d v \text {. }
$$

Substituting Eqs. (3) and (4) into Eq. (6) gives

$$
U_{j}=\frac{1}{2} \int_{l_{j}}^{l_{j+1}}\left\{\begin{array}{l}
\frac{\partial u_{0}(x, t)}{\partial x} \\
\frac{\partial^{2} w_{0}(x, t)}{\partial x^{2}}
\end{array}\right\}^{T}\left[\int_{-\frac{h}{2}}^{\frac{h}{2}}\left[\begin{array}{cc}
E(z) & -z E(z) \\
-z E(z) & z^{2} E(z)
\end{array}\right] d z\right]\left\{\begin{array}{l}
\frac{\partial u_{0}(x, t)}{\partial x} \\
\frac{\partial^{2} w_{0}(x, t)}{\partial x^{2}}
\end{array}\right\} b d x .
$$

In-plane and transverse displacement components within a beam element shown in Fig. 2 are interpolated from element nodal degree of freedom

$$
\begin{aligned}
& u_{0}^{e}(x, t)=N_{1} u_{i}(t)+N_{4} u_{j}(t) . \\
& w_{0}^{e}(x, t)=N_{2} w_{i}(t)+N_{3} \theta_{i}(t)+N_{5} w_{j}(t)+N_{6} \theta_{j}(t) .
\end{aligned}
$$

where shape functions $N_{1}$ and $N_{4}$ are one-dimensional linear Lagrange polynomials, $N_{2}, N_{3}, N_{5}$ and $N_{6}$ are Hermitian interpolation[11].

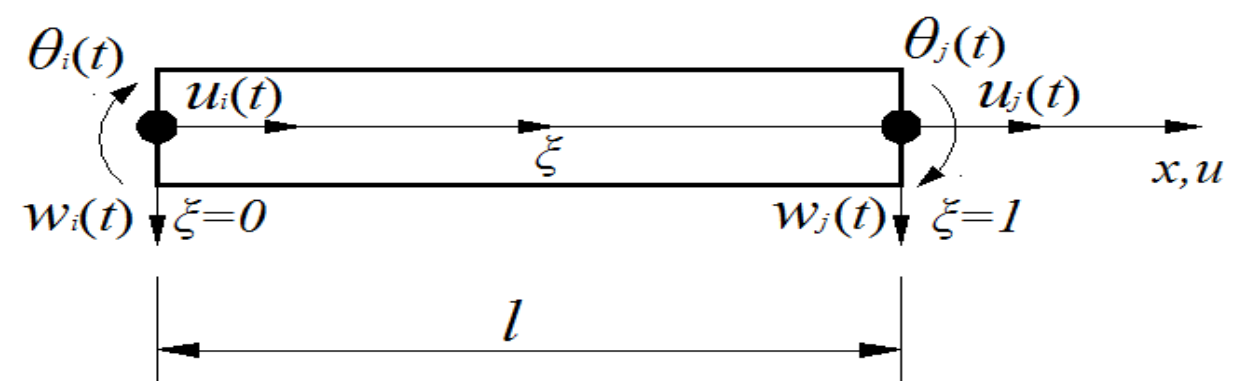

Fig. 2 Two-node beam element with three degrees of freedom per node

Substituting Eqs. (8) and (9) into Eq. (7) yields

$$
U_{j}=\frac{1}{2}\{r(t)\}^{T} \int_{0}^{1}[B]^{T}\left[D_{E}\right][B] b l d \xi\{r(t)\}=\frac{1}{2}\{r(t)\}^{T}\left[K_{j}(E(z))\right]\{r(t)\} .
$$

in which corresponding terms are defined as follows:

The generalized modal parameter $\{r(t)\}=\left\{\begin{array}{llllll}u_{i}(t) & w_{i}(t) & \theta_{i}(t) & u_{j}(t) & w_{j}(t) & \theta_{j}(t)\end{array}\right\}^{T}$.

The $j$ th element stiffness matrix $\left[K_{j}(E(z))\right]=\int_{0}^{1}[B]^{T}\left[D_{E}\right][B] b l d \xi$. where 


$$
\begin{aligned}
& {[B]=\left[\begin{array}{cccccc}
\frac{1}{l} \frac{\partial N_{1}}{\partial \xi} & 0 & 0 & \frac{1}{l} \frac{\partial N_{4}}{\partial \xi} & 0 & 0 \\
0 & \frac{1}{l^{2}} \frac{\partial^{2} N_{2}}{\partial \xi^{2}} & \frac{1}{l^{2}} \frac{\partial^{2} N_{3}}{\partial \xi^{2}} & 0 & \frac{1}{l^{2}} \frac{\partial^{2} N_{5}}{\partial \xi^{2}} & \frac{1}{l^{2}} \frac{\partial^{2} N_{6}}{\partial \xi^{2}}
\end{array}\right] .} \\
& {\left[D_{E}\right]=\int_{-\frac{h}{2}}^{\frac{h}{2}}\left[\begin{array}{cc}
E(\mathbf{z}) & -z E(\mathbf{z}) \\
-z E(\mathbf{z}) & z^{2} E(\mathbf{z})
\end{array}\right] d z .}
\end{aligned}
$$

For the $j$ th element in the $i$ th mode, the EMSE is given as

$$
U_{i j}=\frac{1}{2}\left\{r_{i}(t)\right\}^{T}\left[K_{j}(E(z))\right]\left\{r_{i}(t)\right\} .
$$

where $\left\{r_{i}(t)\right\}$ denotes the generalized modal parameter of the $i$ th mode and can be computed by using the finite element method (FEM) [11]

\section{Damage Indicator Based on Modal Strain Energy Change Ratio}

Based on the finite element model, the MSECRM can be developed to identify the structural damage for the FG Euler-Bernoulli beam. The occurrence of damage in a structure results in a local stiffness reduction and then modifies the mode shapes in a localized region without changing the mass of the structure. From Eq. (15), The EMSE of the $j$ th element in the $i$ th mode before and after the occurrence of damage is given as

$$
\begin{aligned}
U_{i j} & =\frac{1}{2}\left\{r_{i}(t)\right\}^{T}\left[K_{j}(E(z))\right]\left\{r_{i}(t)\right\} . \\
U_{i j}^{d} & =\frac{1}{2}\left\{r_{i}^{d}(t)\right\}^{T}\left[K_{j}{ }^{d}(E(z))\right]\left\{r_{i}^{d}(t)\right\} .
\end{aligned}
$$

where ()$^{d}$ indicates a quantity relating to the damaged states, similarly hereinafter. The location of damage is unknown and therefore the original stiffness matrix approximates the damaged one for the EMSE of damaged state. Eq. (17) can be rewritten as

$$
U_{i j}^{d}=\frac{1}{2}\left\{r_{i}^{d}(t)\right\}^{T}\left[K_{j}(E(z))\right]\left\{r_{i}^{d}(t)\right\} .
$$

The element modal strain energy change is given as

$$
\Delta U_{i j}=\frac{1}{2}\left\{r_{i}^{d}(t)\right\}^{T}\left[K_{j}(E(z))\right]\left\{r_{i}^{d}(t)\right\}-\frac{1}{2}\left\{r_{i}(t)\right\}^{T}\left[K_{j}(E(z))\right]\left\{r_{i}(t)\right\} \text {. }
$$

The modal strain energy change ratio, defined as follows, is chosen to be the damage indicator for the $j$ th element in the $i$ th mode, i.e.,

$$
\zeta_{i j}=\frac{\Delta U_{i j}-\mu_{\Delta U_{i}}}{\sigma_{\Delta U_{i}}} .
$$

where $\mu_{\Delta U_{i}}$ and $\sigma_{\Delta U_{i}}$ denote, respectively, the mean and standard deviation of $\Delta U_{i j}$, i.e.,

$$
\mu_{\Delta U_{i}}=\frac{1}{n} \sum_{j=1}^{n} \Delta U_{i j} \text { and } \sigma_{\Delta U_{i}}=\sqrt{\frac{\sum_{j=1}^{n}\left(\Delta U_{i j}-\mu_{\Delta U_{i}}\right)^{2}}{n-1}} \text {. }
$$

where $n$ is the number of elements. If the $\zeta_{i j}$ for several modes are considered together, the damage indicator of the $j$ th element is defined as the mean of $\zeta_{i j}$ for a set of first few lower mode shapes, i.e.,

$$
\zeta_{j}=\frac{1}{m} \sum_{i=1}^{m} \zeta_{i j}=\frac{1}{m} \sum_{i=1}^{m} \frac{\Delta U_{i j}-\mu_{\Delta U_{i}}}{\sigma_{\Delta U_{i}}}
$$

where $\zeta_{j}$ represents the damage indicator of the jth element, $m$ is the number of mode shapes. In this study, only the three first lower mode shapes are required since the contribution of the higher mode 
shapes are insignificant[6]. The largest value of $\zeta_{j}$ means potential damage location in the FG Euler-Bernoulli beam.

\section{Numerical Example}

A numerical example is presented to test the validity of the proposed method. The example involves an FG Euler-Bernoulli simply-supported beam of length $L=5 \mathrm{~m}$, width $b=0.1 \mathrm{~m}$, thickness $h=0.2 \mathrm{~m}$, as shown in Fig. 3. The finite element model of the beam consists of 10 elements (2-node beam element with 3 degrees of freedom per node). The material properties of the FGM constituents[12] are listed in Table 1 . Young's modulus $E$ and mass density $\rho$ vary according to Eq. (1). Element 2 is assumed as the damaged element with different levels of damage (its stiffness reduced by $4 \%$ and $10 \%$, respectively). Four cases of the power-law exponent $k$, which take the values of $0,1,5$ and 10, are considered. All the finite element analysis is done using ABAQUS standard finite element program.

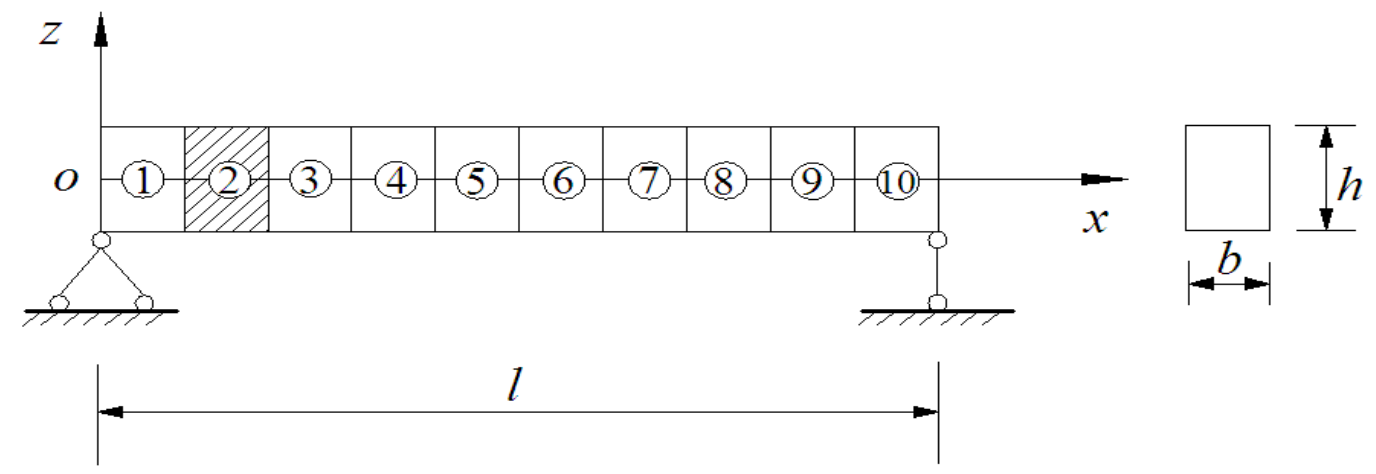

Fig. 3 The element model of damage identification of the FG Euler-Bernoulli simply-supported beam

Table 1 Material properties of FGM constituents

\begin{tabular}{ccc}
\hline Properties & $\begin{array}{c}\text { Lower surface } \\
(\text { Steel })\end{array}$ & $\begin{array}{c}\text { Upper surface } \\
\left(\text { Alumina, } \mathrm{Al}_{2} \mathrm{O}_{3}\right)\end{array}$ \\
\hline $\mathrm{E}(\mathrm{GPa})$ & 210 & 390 \\
$\rho\left(\mathrm{kg} / \mathrm{m}^{3}\right)$ & 7800 & 3960 \\
\hline
\end{tabular}

Instead of carrying out actual experiment, the generalized modal parameter are simulated using the FEM. In the practical measurement, the noises are unavoidable. The FEM-computed generalized modal parameter is directly added a Gauss noise for the simulation of the noise contamination. Hence, the generalized modal parameter with noise contamination is defined as

$$
\bar{r}_{i j}=r_{i j}\left(1+\eta_{i} p^{e}\right) \text {. }
$$

where $\bar{r}_{i j}$ is the $i$ th generalized modal parameter in the $j$ th mode with noise contamination, $r_{i j}$ is the $i$ th generalized modal parameter in the $j$ th mode with no noise, $\eta_{i}$ is a random number generated from a Gauss distribution with mean 0 and standard deviation $1, p^{e}$ denotes the level of noise and take the values of $0 \%, 1 \%, 2 \%$ and $5 \%$.

The damage identification results are shown in Figs. 4 and 5. For four cases of the power-law exponent $k$, the largest values of the damage indicator $\zeta_{j}$ are found in element 2 with any levels of damage and noise. For the other undamaged elements, all the values of the damage indicator $\zeta_{j}$ are much less than the corresponding ones of element 2. It means that the damaged element can be effectively detected by using the damage indicator $\zeta_{j}$. The numerical example shows that the proposed method has very good capability for identifying damage in the FG Euler-Bernoulli beam and is robust to the effects of measurement noise. 


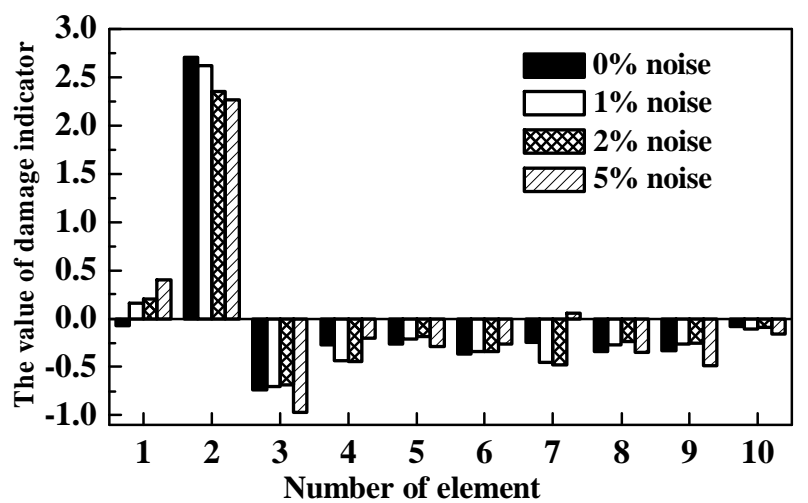

(a) power-law exponent $k=0$

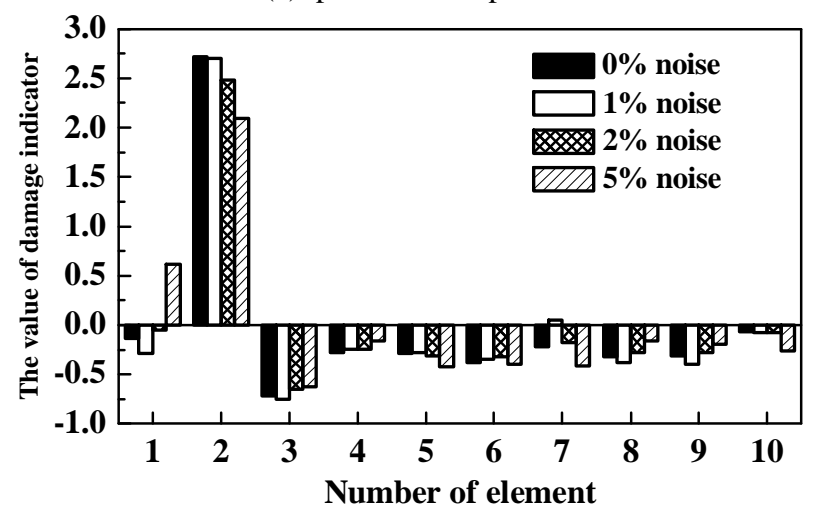

(c) power-law exponent $k=5$

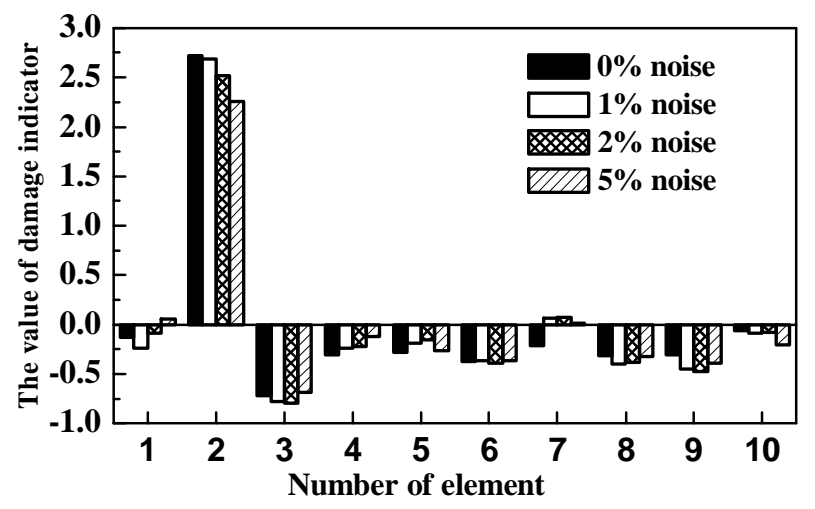

(b) power-law exponent $k=1$

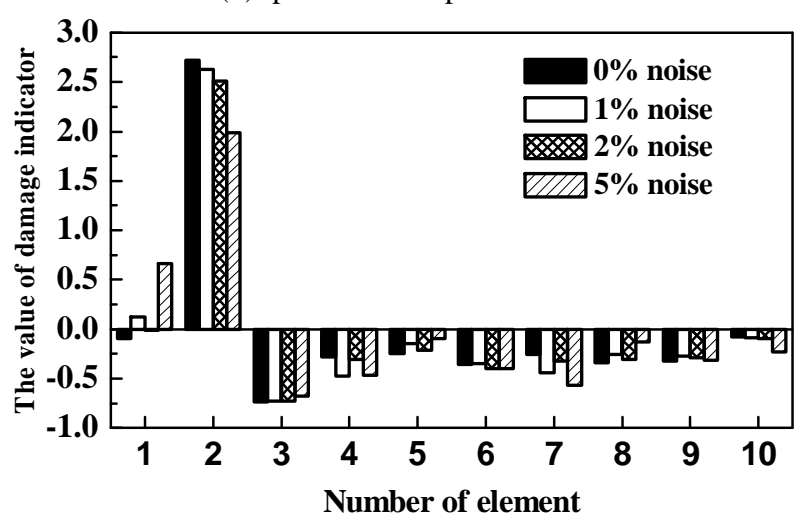

(d) power-law exponent $k=10$

Fig. 4 Damage identification results of FG Euler-Bernoulli beam with damage in element 2 (stiffness reduced by $4 \%$ )
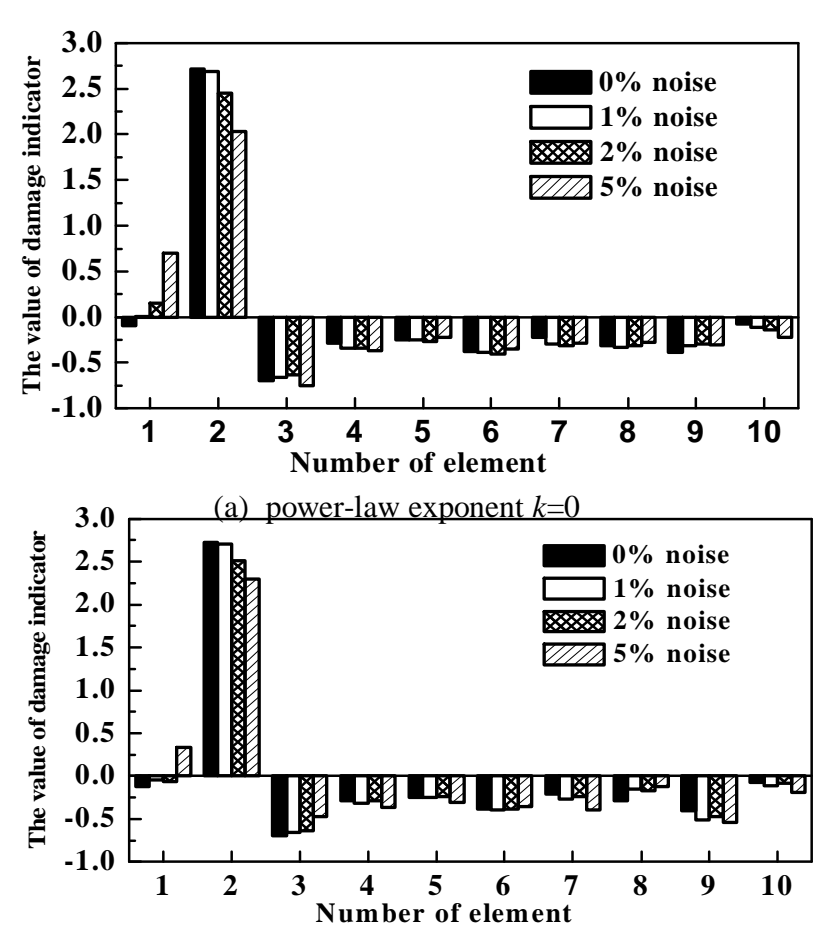

(c) power-law exponent $k=5$

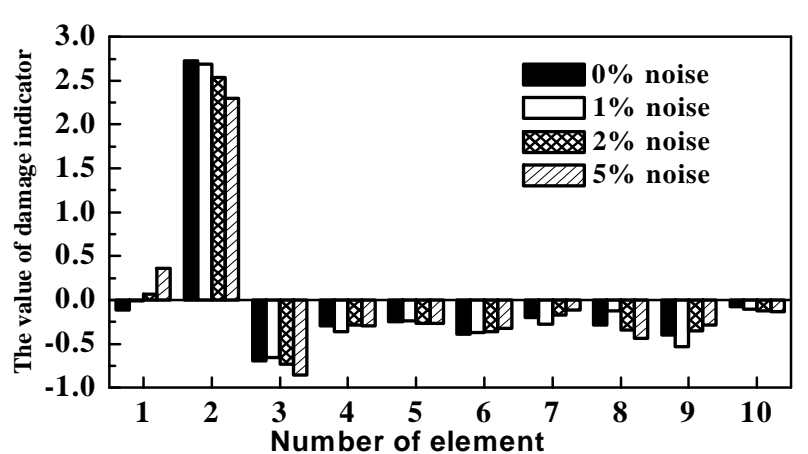

(b) power-law exponent $k=1$

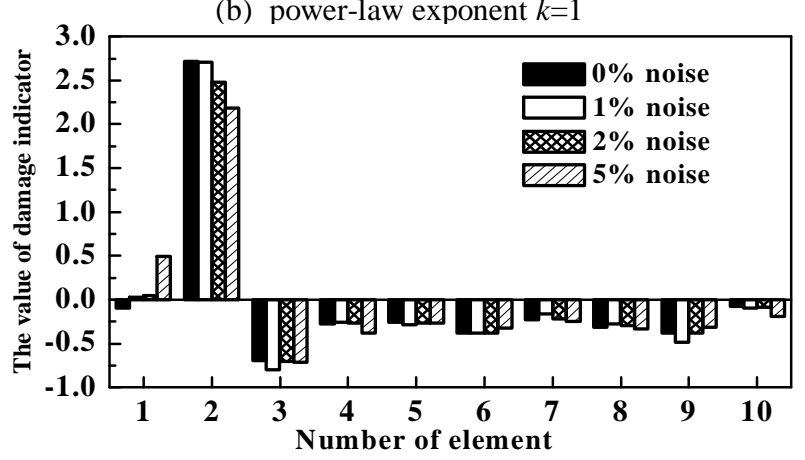

(d) power-law exponent $k=10$

Fig. 5 Damage identification results of FG Euler-Bernoulli beam with damage in element 2 (stiffness reduced by $10 \%$ ) 


\section{Summary}

An identification method based on MSE has been proposed in this paper for identifying the damage of the FG Euler-Bernoulli beam. From the strain energy of the FG Euler-Bernoulli beam, the expression of EMSE is developed for the two-node beam element with three degrees of freedom per node. ABAQUS standard finite element program is used to compute the element stiffness matrix and the generalized modal parameters for the EMSE. Based on the change of the EMSE before and after the occurrence of damage, modal strain energy change ratio is used as the damage indicator for the damage identification of the FG Euler-Bernoulli beam. The validity of the proposed method is illustrated by a numerical example. The numerical results demonstrate that the proposed method has very good capability for the damage identification of the FG Euler-Bernoulli beam. Furthermore, the unavoidable noise in measurement is modeled by Gaussian noise. With the noise-contaminated measurement, the damaged element can be still detected easily. Those numerical results reveal that the proposed method is robust to the effects of the measured modal parameters noise.

\section{Acknowledgements}

This work is financially supported by the National Natural Science Foundation of China (11262002) and those supports are gratefully acknowledged.

\section{References}

[1] J. V. Araújo dos Santos, C. M. Mota Soares, C. A. Mota Soares, H. L. G. Pina, Development of a numerical model for the damage identification on composite plate structures, Compos. Struct. 48(2000) 59-65.

[2] S. W. Doebling, C. R. Farrar, M. B. Prime, and D. W. Shevitz, Technical Report LA-13070-MS, New Mexico, USA, 1996, Los Alamos National Laboratory, Los Alamos.

[3] L. H. Yam, T. P. Leung, D. B. Li, K. Z. Xue, Theoretical and experimental study of modal strain analysis, J. Sound Vib. 191(1996) 251-260.

[4] J.M.Ndambi, J.Vantomme, K.Harri, Damage assessment in reinforced concrete beams using eigenfrequencies and mode shape derivatives, Eng. Struct. 24(2002) 501-515.

[5] P.J. Cornwell, S.W. Doebling, C.R. Farrar, Application of the strain energy damage detection method to plate-like structures, J. Sound Vib. 224(1999) 359-374.

[6] Z.Y. Shi, S. S. Law, L. M. Zhang, Structural damage localization from modal strain energy change, J. Sound Vib. 218(1998) 825-844.

[7] Z.Y. Shi, S. S. Law, L. M. Zhang, Structural damage detection from modal strain energy change, J. Eng. Mech. 126(2000) 1216-1223.

[8] Y.Y. Li, Hyper sensitivity of strain-based indicators for structural damage identification: A review, Mechanical Syst. Sign. Process. 24(2010) 653-664.

[9] A. Alvandia, C. Cremona, Assessment of vibration-based damage identification techniques, J. Sound Vib. 292(2006) 179-202.

[10] M. Simsek, T. Kocaturk, Free and forced vibration of a functionally graded beam subjected to a concentrated moving harmonic load, Compos. Struct. 90(2009) 465-473.

[11]R. D. Cook, D. S. Malkus, M. E. Plesha, Concepts and applications of finite element analysis, third ed., John Wiley \& Sons, Inc., Toronto, Canada, 1989.

[12] A. E. Alshorbagy, M. A. Eltaher, F. F. Mahmoud, Free vibration characteristics of a functionally graded beam by finite element method, Appl. Math. Modelling, 35(2011) 412-425. 